\title{
Optimal calculating on working point of the permanent magnet
}

\author{
Yanli Gao ${ }^{1, \text { a }}$, Jianming Huang ${ }^{2}$ and Shiyong Zhang ${ }^{2}$ \\ ${ }^{1}$ Naval Aeronautical Engineering Institute Qingdao Branch \\ ${ }^{2}$ Unit 92557 of PLA \\ agaoyanli2003@163.com
}

Keywords: Permanent magnet; Working point; Optimal calculating; Magnetic energy density ; Polarized system

\begin{abstract}
Confirming about the working point of the permanent magnet is an essential premise in the calculating of permanent magnetic circuit. In this paper, the choosing methods about the working point of the permanent magnet are analyzed overall. The calculating formulas of the optimal calculating are deduced using the conception of magnetic energy density. The conclusions can give some guidance to the permanent magnetic circuit calculating and design. The example of calculating work points of permanent magnet for the typical polarized systems are proposed.
\end{abstract}

\section{Introduction}

With the wide application of permanent magnetic material, the calculating of permanent magnetic circuit is more and more important. The choosing of working point of permanent magnetic is the base of calculating of permanent magnetic circuit. Permanent magnet is working based on remanence of magnetic material, and has much larger coercive force. In the analysis of permanent magnetic circuit, the permanent magnet is corresponding as a magneto-motive force and provides with magnetic resistance. There are two kinds of working states, one kind is that the permanent magnet works at demagnetization curve and magnetic resistance keeps constant, the other is permanent magnet works at reverting line and magnetic resistance is variable[1-5]. The optimal calculating of permanent magnet working point can improve the efficiency of magnetic circuit[6-8]. In this paper, the working points of the two kinds of working state for permanent magnet are analyzed, and some correlative calculating formulae are deduced. The example of calculating work points of permanent magnet and air magnetic flux for the typical polarized systems are proposed.

\section{Optimal calculation of permanent magnet working point}

The working point of permanent magnet has connection with technique of magnetization. Because of the lower magneto-conductivity of permanent magnet and the much influence of leakage magnetic flux, the leakage magnetic flux phenomenon must be considered. The permanent magnetic circuit model is shown as Fig.1.

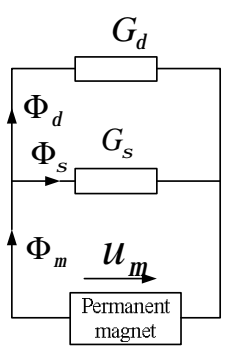

Fig.1 Model of permanent magnetic circuit

The leakage magnetic coefficient is defined as

$$
\sigma=\Phi_{\mu} / \Phi_{\delta}=b_{\mu} / b_{\delta \mu}
$$


where,$\Phi_{\mu}$ is main magnetic flux, $\Phi_{\delta}$ is air gap magnetic flux, $\Phi_{\sigma}$ is leakage magnetic flux, $b_{\mu}$ is magnetic induction intensity, $b_{\delta \mu}$ is converting air gap magnetic induction intensity, $G_{\delta}$ is air gap magneto conductivity and $G_{\sigma}$ is leakage magneto conductivity.

Magnetic field energy of air gap is

$$
\begin{aligned}
A_{\delta} & =0.5 \Phi_{\delta} \mu_{\delta}=0.5\left(\frac{b_{\mu}}{\sigma} S_{\mu}\right)\left(-H_{\mu} L_{\mu}\right) \\
& =-0.5 B_{r} H_{c}\left[-\frac{1}{\sigma} \frac{b_{\mu}}{B_{r}}\left(-\frac{H_{\mu}}{-H_{c}}\right)\right] V_{\mu} \\
& =-0.5 B_{r} H_{c}\left(-\frac{1}{\sigma} b h\right) V_{\mu}=-\frac{B_{r} H_{c}}{2} w_{\delta} V_{\mu}
\end{aligned}
$$

Where, $w_{\delta}=-b h / \sigma$ is converting magnetic energy density. $b=b_{\mu} / B_{r}, h=-H_{\mu} / H_{c}, B_{r}$ and $H_{c}$ are remanence and coercive force of permanent magnetic material. $H_{\mu}$ is permanent magnetic field intensity.

\section{Calculating of working point at demagnetization curve}

When permanent magnet is magnetized at the state of complete assembly and no demagnetizing effect occurs, the permanent magnet works at demagnetization curve. When working point changes, the leakage magnetic coefficient $\sigma$ changes slightly and $\sigma$ can be approximately constant. Then, $\sigma=b_{\mu} / b_{\delta}$ and air magnetic induction intensity $b_{\delta}=b_{\mu}-b_{\sigma}$. The converting air gap magnetic energy density is

$$
w_{\delta}=-b_{\delta} h_{\mu}=-\frac{b_{\mu}}{\sigma} h_{\mu}
$$

According to experiential formula of demagnetization curve, then

$$
b_{\mu}=\frac{1+h_{\mu}}{1+a h_{\mu}}, a=\frac{2 \sqrt{r}-1}{r}
$$

where $r$ is bulge coefficient of magnetic material.

Thus

$$
w_{\delta}=-\frac{h_{\mu}\left(1+h_{\mu}\right)}{\sigma\left(1+a h_{\mu}\right)}
$$

The extremum condition of $w_{\delta}$ is $d w_{\delta} / d h_{\mu}=0$, then

$$
\left\{\begin{array}{c}
h_{\mu m}=\frac{1}{\alpha}(\sqrt{1-\alpha}-1) \\
b_{\mu m}=\frac{1+h_{\mu m}}{1+\alpha h_{\mu m}}=\frac{1-\sqrt{1-\alpha}}{\alpha}
\end{array}\right.
$$

Thus, the slope of line between working point of permanent magnet and coordinate origin is

$$
\mu_{*}=\frac{b_{\mu m}}{h_{\mu m}}=\frac{b_{\delta m}+b_{\sigma m}}{h_{\mu m}}=\mu_{\delta^{*}}+\mu_{\sigma^{*}}
$$

or

$$
\mu_{*}=B_{r} / H_{c}
$$

The max magnetic energy density is

$$
w_{\delta m}=-\frac{b_{\mu m} h_{\mu m}}{\sigma}=\frac{2(1-\sqrt{1-\alpha})-\alpha}{\sigma \alpha^{2}}
$$

Therefore, the conclusion can be achieved: the optimal working point of permanent magnet is point of intersection between the line of coordinate origin and $A\left(B_{r},-H_{c}\right)$ and demagnetization curve when 
permanent magnet works at demagnetization curve. The conclusion of equation (5) to (7) is denoted as Fig.2

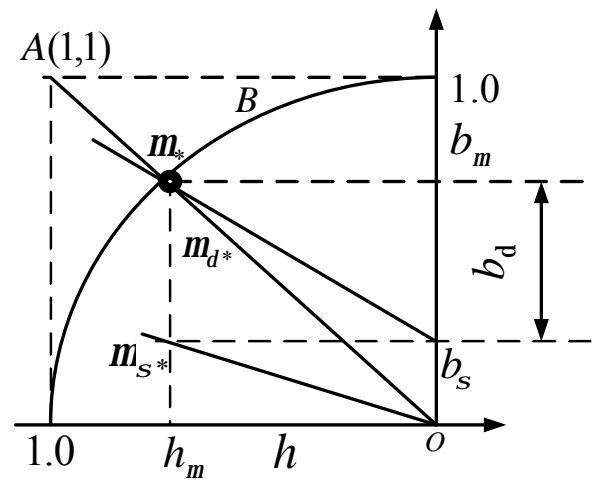

Fig.2 Permanent magnet works at demagnetization curve

\section{Calculating of working point at reverting line}

In order to keep the working point of permanent magnet from the affected by outside magnetic field, some AC magnetic field is needed to demagnetize a certain extent and make the permanent magnet works at a steady reverting line. Two kinds are classified : one kind is magnetized at the state of assembling partially and its leakage magneto conductivity. is equal to that of assembling completely, namely that $\mu_{\sigma^{*}}=\mu_{\sigma^{*} 0}$. The other is magnetized at the state of assembling partially and its leakage magneto conductivity. is not equal to that of assembling completely, namely that $\mu_{\sigma^{*}} \neq \mu_{\sigma^{*} 0}$. The confirming of working point is determined by the intensity of outside interferential magnetic field, and the working point of permanent magnet should not transfer to demagnetization curve in the instance of intense outside interferential magnetic field. Otherwise, the start point of reverting line will change when outside interferential magnetic field is wiped off and the B value of working point will decrease.

1. The optimal working point of $\mu_{\sigma^{*}}=\mu_{\sigma^{*} 0}$. Supposing the start of reverting line is $A_{1}\left(h_{1}, b_{1}\right)$ and working point is $A_{2}\left(h_{2}, b_{2}\right)$, as fig.3. Leakage magnetic coefficient is given at assembling partially

$$
\sigma=b_{1} / b_{\delta 0}
$$

Where $b_{\delta 0}$ is air gap magnetic induction intensity, $b_{1}=b_{\delta 0}+b_{\sigma}, b_{\sigma}$ is leakage magnetic induction intensity, shown as Fig.3.

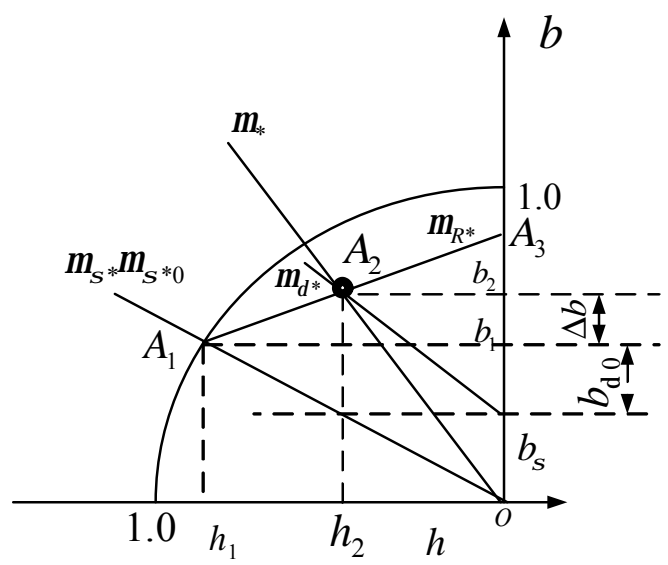

Fig.3 Working at reverting line $\left(\mu_{\sigma^{*}}=\mu_{\sigma^{*} 0}\right)$

According to engineering experiential formula, equivalent expressions of demagnetization curve is $b=(1+h) /(1+a h)$, reverting coefficient $\mu_{R^{*}}=1-\alpha$.

When working point transfers from $A_{1}\left(h_{1}, b_{1}\right)$ to $A_{2}\left(h_{2}, b_{2}\right)$, air magnetic induction intensity is

$$
\begin{aligned}
b_{\delta} & =b_{\delta 0}+\Delta b=b_{1} / \sigma+\mu_{R^{*}}\left(h_{2}-h_{1}\right) \\
& =\frac{1+h_{1}}{\sigma\left(1+a h_{1}\right)}+(1-\alpha)\left(h_{2}-h_{1}\right)
\end{aligned}
$$


The converting magnetic energy density of air gap is

$$
w_{\delta}=-h_{2} b_{\delta}=-h_{2}(1-\alpha)\left(h_{2}-h_{1}\right)-h_{2} \frac{1+h_{1}}{\sigma\left(1+\alpha h_{1}\right)}
$$

Where $-h_{2} b_{\delta 0}=-h_{2} \frac{1+h_{1}}{\sigma\left(1+\alpha h_{1}\right)}$ is convert-ing value of magnetic energy density with starting air gap of working point. $-h_{2}(1-\alpha)\left(h_{2}-h_{1}\right)$ is converting value of magnetic energy density increment from assembling partially to assembling totally.

From geometrical connection of Fig. 3, if $A_{1}\left(h_{1}, b_{1}\right)$ is decided, $\mu_{R^{*}}$ will be decided. The conduction of the max inner connected rectangle area of $\Delta O A_{1} A_{3}$ is $h_{2}=0.5 h_{1}$. To meet this conduction, $b_{\sigma}=0.5 b_{1}$ and leakage magnetic coefficient $\sigma=b_{1} / b_{\delta 0}=2$, then converting value of air gap

magnetic energy density is :

$$
w_{\delta}=-\frac{h_{1}}{2}\left[(1-\alpha) \cdot 0.5 h_{1}+\frac{1+h_{1}}{2\left(1+\alpha h_{1}\right)}\right]
$$

Obviously, the location of $A_{1}\left(h_{1}, b_{1}\right)$ has influence on $w_{\delta}$ and there is a $h_{1 m}$ value to maximize $w_{\delta}$. If the method of derivate to $w_{\delta}$ is used to solve the $h_{1 m}$, a cubic equation will be got and difficult to solve. If $\alpha$ is given and draws the chart of $w_{\delta}=f\left(h_{1}\right)$, it is convenient to find $h_{1 m}$.

In the actual engineering, $\sigma$ is always bigger than 2 , the change of $b_{2}$ is very little and approaches to $b_{m}$ for different $\alpha$ value. The optimal working point is solved as

$$
\left\{\begin{array}{c}
b_{2}=\frac{1-\sqrt{1-\alpha}}{\alpha} \\
b_{1}=\frac{1+h_{1}}{1+\alpha h_{1}} \\
\frac{b_{2}}{b_{1}}=\frac{h_{2} \mu_{*}}{h_{1} \mu_{\sigma^{*}}}=\frac{h_{2}}{h_{1}} \frac{\sigma}{\sigma-1}
\end{array}\right.
$$

2. The optimal working point of $\mu_{\sigma^{*}} \neq \mu_{\sigma^{*} 0}$. Making the coefficient $k_{y}=\mu_{\sigma^{*}} / \mu_{\sigma^{*} 0}$ and leakage magnetic coefficient $\sigma=b_{1} / b_{\delta 0}$, the $w_{\delta}$ is not dicided by inner connected rectangle area of $\Delta O A_{1} A_{3}$ and a apex is lies at $\mu_{\sigma^{*}}$ line, as Fig.4.

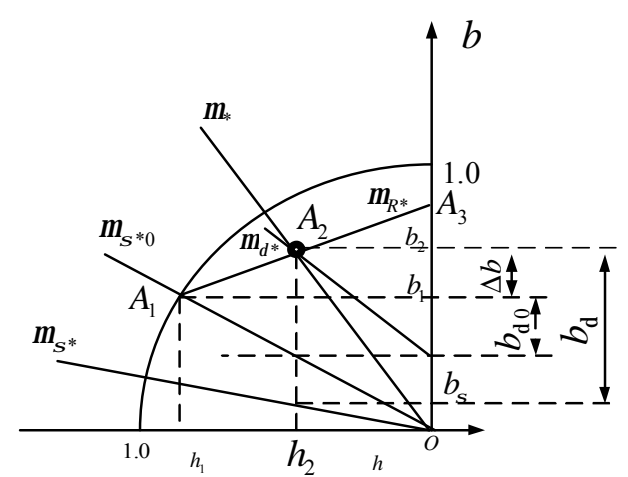

Fig.4 Working at reverting line $\left(\mu_{\sigma^{*}} \neq \mu_{\sigma^{*} 0}\right)$

From fig. 4 , it is difficult to get

$\left\{\begin{array}{c}\mu_{\delta^{*}}=b_{\sigma} / h_{2} \\ \mu_{\sigma^{* 0} 0}=\left(b_{1}-b_{\delta 0}\right) / 2\end{array}\right.$

then $k_{y}=\mu_{\sigma^{*}} / \mu_{\sigma^{*} 0}=b_{\sigma} /\left(b_{1}-b_{\delta 0}\right)$,

Accordingly 
$b_{\sigma}=k_{y}\left(b_{1}-b_{\delta 0}\right)=k_{y} b_{1}[(\sigma-1) / \sigma]$

and

$$
b_{\delta}=b_{2}-b_{\sigma}=b_{1}-b_{\sigma}+\Delta b
$$

Therefore

$$
b_{\delta}=b_{1}\left(1-k_{y} \frac{\sigma-1}{\sigma}\right)+(1-\alpha)\left(h_{2}-h_{1}\right)
$$

Making $\sigma_{p}=\frac{\sigma}{\sigma-k_{y}(\sigma-1)}$, then

$$
b_{\delta}=b_{1} / \sigma_{p}+(1-\alpha)\left(h_{2}-h_{1}\right)=b_{1} / \sigma_{p}+\mu_{R^{*}}\left(h_{2}-h_{1}\right)
$$

Equation (18) is similar with (10), the difference is $\sigma_{p}$ and $\sigma$.

\section{Conclusions}

The optimal calculating of permanent magnet working point are presented under different working conditions. The calculating equations are applicable to nonlinear demagnetization curve of permanent magnet, such as AlNiCo. Moreover, for the thulium permanent magnet, the calculating method is much simple because of their line demagnetization curve. The example of calculating work points of permanent magnet and air magnetic flux for the typical polarized systems are proposed .

\section{References}

[1] Tong Weiming, Zai Guofu. Low voltage electrical equipment relay and control system. Harbin: Harbin industry institute press, 2000

[2] Liu Lingshun. Analysis and calculation of permanent magnetic working points of polarity magnetic system, Journal of naval aeronautical and astronautical university, Vol 5,Issue 17, 2002.pp.183-185

[3] Tong Weiming, Li Fengge,Zhao Zhiheng. Diagrammatized method unified model and analysizing of the permanent magnet's working point in the polarized mgnetic system with single permanent magnet. Proceedings of the CSEE. Vol 6,Issue 25,2005.pp.129-133

[4] Tong Weiming, Liang Huimin, Li Fengge, et al. Reasonable criterion and its application of polarized magnetic system, Proceedings of the CSEE. Vol.3,Issue 22,2002.pp.83-87

[5] Zhai Guofu, Liang Huimin, Guo Chenghua.Research and analysis on the shape of the permanent magnet torque characteristic curve of polarized magnetic system. Proceedings of the CSEE. Vol 11, Issue22, 2002.pp.110-114

[6] Shoffa V N, Chichervukin V N , Ivakin B F . A mathematical model and a procedure for designing the complex magnetic system of a polarized relay. Elecktrichestvo.Vol 7. 2004.pp.50-55 .

[7] Liang Huimin, Zhai Guofu, Luo Fubiao . Virtual design of permanent magnet reversion line's starting point in polarized magnetic system based on finite element. Proceeding of the CSEE.Vol.9. Issue 25.2005.pp. 147-152 .

[8] Zhai Guofu, Liang Huimin,Wang Hao,et al . Research on the parameters optimum design of polarized magnetic system based on orthogonal design . Proceeding of the CSEE , Vol0, Issue 23.2003.pp.158-163 